\title{
Differential regulation of synaptic vesicle tethering and docking by UNC-18 andTOM-1
}

\author{
' Department of Physiology, University of California at San Francisco, USA. \\ 2 Department of Molecular Genetics and Cell Biology, University of Illinois at Chicago, IL, USA \\ ${ }^{3}$ Center for Integrative Genomics, University of Lausanne, Lausanne, Switzerland \\ ${ }^{4}$ Department of Biological Sciences, University of Illinois at Chicago, IL, USA
}

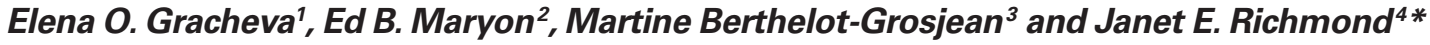

\section{Edited by:}

Robert Renden,

UCB Pharma SA, Belgium

Reviewed by:

Thomas Söllner,

University of Heidelberg, Germany

Susanne Schoch,

University of Bonn, Germany

${ }^{*}$ Correspondence:

Janet E. Richmond,

Department of Biological Sciences,

University of Illinois at Chicago, 840

West Taylor Street Chicago, IL 60607,

USA.

e-mail: jer@uic.edu
The assembly of SNARE complexes between syntaxin, SNAP-25 and synaptobrevin is required to prime synaptic vesicles for fusion. Since Munc18 and tomosyn compete for syntaxin interactions, the interplay between these proteins is predicted to be important in regulating synaptic transmission. We explored this possibility, by examining genetic interactions between $C$. elegans unc-18(Munc18), unc-64(syntaxin) and tom-1(tomosyn). We have previously demonstrated that unc-18mutants have reduced synaptic transmission, whereas tom-1 mutants exhibit enhanced release. Here we show that the unc-18 mutant release defect is associated with loss of two morphologically distinct vesicle pools; those tethered within $25 \mathrm{~nm}$ of the plasma membrane and those docked with the plasma membrane. In contrast, priming defective unc-13 mutants accumulate tethered vesicles, while docked vesicles are greatly reduced, indicating tethering is UNC-18-dependent and occurs in the absence of priming. C. elegans unc-64 mutants phenocopy unc-18 mutants, losing both tethered and docked vesicles, whereas overexpression of open syntaxin preferentially increases vesicle docking, suggesting UNC-18/closed syntaxin interactions are responsible for vesicle tethering. Given the competition between vertebrate tomosyn and Munc18, for syntaxin binding, we hypothesized that $C$. elegansTOM-1 may inhibit both UNC18-dependent vesicle targeting steps. Consistent with this hypothesis, tom-1 mutants exhibit enhanced UNC-18 plasma membrane localization and a concomitant increase in both tethered and docked synaptic vesicles. Furthermore, in tom-1; unc-18 double mutants the docked, primed vesicle pool is preferentially rescued relative to unc-18 single mutants. Together these data provide evidence for the differential regulation of two vesicle targeting steps by UNC-18 and TOM-1 through competitive interactions with syntaxin.

Keywords: Caenorhabditis elegans, tomosyn, vesicle docking, vesicle tethering, syntaxin, UNC-18, synaptic transmission, high-pressure freeze electron microscopy

\section{INTRODUCTION}

Rapid, calcium-regulated release of neurotransmitter is dependent on the availability of a readily releasable, primed synaptic vesicle pool (Sudhof, 2004). The priming process that renders this pool fusion competent requires the assembly of soluble $\mathrm{N}$-ethylmaleimidesensitive factor attachment protein receptor (SNARE) complexes composed of the vesicle SNARE, synaptobrevin and the plasma membrane SNAREs, syntaxin and SNAP-25 (Jahn and Scheller, 2006). Formation of the SNARE complex is regulated by several highly conserved proteins including; Munc13, the sec-1/Munc18 (SM) protein family and tomosyn, all of which are known to bind syntaxin. Despite a growing consensus on the regulatory functions performed by these proteins, there remain some inconsistencies in the interpretation of precisely which stages of vesicle maturation are impacted, as outlined below.

It is well-established that Munc13 proteins play a critical role in priming, null mutants in mouse Munc13-1/2 (Augustin et al., 1999; Varoqueaux et al., 2002). Drosophila Dunc-13 (Aravamudan et al., 1999) and C. elegans UNC-13 (Richmond et al., 1999) exhibiting a profound loss of fusion competent synaptic vesicles. Current models suggest that Munc13s promote SNARE complex assembly and stability, through interactions with syntaxin (Betz et al., 1997; Richmond et al., 2001; Basu et al., 2005; Madison et al., 2005; Stevens et al., 2005). This function of Munc13 was initially attributed to a post vesicle docking stage, on the basis of ultrastructural data from aldehyde fixed tissues which showed normal docking in null mutants (Aravamudan et al., 1999; Richmond et al., 1999; Varoqueaux et al., 2002). However, recent implementation of high-pressure freeze (HPF) fixation methods and ultrastructural analyses have demonstrated that the priming defects of both Munc13 and unc-13 mutants are associated with a pronounced reduction in plasma membrane docked synaptic vesicles at the active zone (defined as $0 \mathrm{~nm}$ between vesicle and plasma membranes) (Weimer et al., 2006; Siksou et al., 2009). These contradictory results can be explained by differences in fixation methods (Siksou et al., 2007). That this recently revealed docking defect reflects the loss of assembled SNARE complexes in Munc13/unc-13 mutants is supported by the observation that $C$. elegans syntaxin null mutants prepared by HPF fixation exhibit a similar docking defect (Hammarlund et al., 2007).

Prior to this HPF data, docking was thought to occur upstream of vesicle priming and to involve members of the SM protein family. The docking role of Munc18/UNC-18, based on aldehyde fixed 
electron micrographs, show reduced membrane-associated vesicles at C. elegans unc-18 mutant synapses and chromaffin cells isolated from Munc18-1 mutant mice (Voets et al., 2001; Weimer et al., 2003). More recent live imaging data using total internal reflection fluorescence microscopy (TIRFM), suggests that Munc18 actually impacts several kinetically distinct events in the movements and dwell times of dense core vesicles relative to the plasma membrane (Toonen et al., 2006). These likely reflect some of the different functional roles attributed to SM proteins at progressive stages of secretory vesicle maturation including; the chaperoning of syntaxin to the membrane (Rowe et al., 2001; McEwen and Kaplan, 2008), the stabilization of syntaxin/SNAP-25 dimers in preparation for SNARE complex assembly (Zilly et al., 2006; de Wit et al., 2009), the docking of vesicles prior to priming (Voets et al., 2001; Weimer et al., 2003; Gulyas-Kovacs et al., 2007) and the promotion of fusion competent vesicles in conjunction with the assembled SNARE complex (Dulubova et al., 2007; GulyasKovacs et al., 2007; Shen et al., 2007). Therefore, it seems likely that reevaluation of Munc18/UNC-18 ultrastructure using HPF fixation techniques could reveal previously unidentified changes in vesicle targeting reflecting these multifaceted roles in exocytosis.

Tomosyn is known to compete with Munc18 for syntaxin binding both in vitro (Fujita et al., 1998) and in situ (Gladycheva et al., 2007). Through a C-terminal SNARE binding motif, tomosyn also forms a SNARE complex with syntaxin and SNAP-25, excluding synaptobrevin (Hatsuzawa et al., 2003; Pobbati et al., 2004). As a result, the formation of tomosyn SNARE complexes has been shown to inhibit vesicle priming and negatively regulate neurosecretion (Yizhar et al., 2004; Gracheva et al., 2006, 2007; McEwen et al., 2006; Sakisaka et al., 2008). Consequently, loss of tomosyn at C. elegans tomosyn (TOM-1) mutant synapses results in increased vesicle priming and a concomitant increase in plasma membrane contacting synaptic vesicles based on HPF fixation (Gracheva et al., 2006).

Given that the opposing functions of Munc18 and tomosyn in the maturation of primed synaptic vesicles are dependent on competition for syntaxin binding, we hypothesized that analyzing genetic interactions between these three proteins would provide new insights into the specific vesicle trafficking steps that they regulate. We addressed this hypothesis by examining the functional interplay between unc-18, unc-64 and tom-1 in intact C. elegans mutant synapses using a combination of HPF ultrastructural and electrophysiological analyses.

\section{MATERIALS AND METHODS GENETICS}

Nematode strains were maintained at $20-25^{\circ} \mathrm{C}$ on agar plates seeded with OP50 bacteria using standard methods, as described previously (Brenner, 1974). The strains used were: the wild-type Bristol N2 isolate, unc-18(b403) and RM299, unc-18(md299), VC223, tom-1(ok285), SY1064, tom-1(ok285);unc-18(b403), SY1424, tom-1(ok285);unc18(md299), EG3278, unc-64(js115) and oxEx536[Punc17:SYX;Pglr-1:SYX; Punc-122:GFP; lin-15(+)]; BC168, unc-13(s69), EG1985, unc-64(js115);oxIs34(UNC-64LE166/167AA).

\section{ELECTROPHYSIOLOGY}

Electrophysiological methods were as previously described (Richmond, 2009). Briefly, animals were immobilized with Histoacryl Blue glue, and a lateral cuticle incision was made with a glass needle, exposing the ventral medial body wall muscles. Body wall muscle recordings were made in the whole-cell voltage-clamp configuration (holding potential, $-60 \mathrm{mV}$ ) using an EPC-10 patch-clamp amplifier and digitized at $1 \mathrm{kHz}$. The extracellular solution consisted of $150 \mathrm{mM} \mathrm{NaCl}, 5 \mathrm{mM} \mathrm{KCl}$, $5 \mathrm{mM} \mathrm{CaCl}_{2}, 4 \mathrm{mM} \mathrm{MgCl}, 10 \mathrm{mM}$ glucose, $5 \mathrm{mM}$ sucrose, and $15 \mathrm{mM}$ HEPES ( $\mathrm{pH} 7.3, \sim 340 \mathrm{mOsm}$ ). The patch pipette was filled with $120 \mathrm{mM} \mathrm{KCl}, 20 \mathrm{mM} \mathrm{KOH}, 4 \mathrm{mM} \mathrm{MgCl}_{2}, 5 \mathrm{mM}$ [N-tris(Hydroxymethyl) methyl-2-aminoethane-sulfonic acid), $0.25 \mathrm{mM} \mathrm{CaCl}_{2}, 4 \mathrm{mM} \mathrm{Na}$ ATP, $36 \mathrm{mM}$ sucrose, and $5 \mathrm{mM}$ EGTA ( $\mathrm{pH} 7.2, \sim 315 \mathrm{mOsm})$. Hyperosmotic responses were recorded using extracellular solution adjusted to $800 \mathrm{mOsm}$ with sucrose. Data were acquired using Pulse software (HEKA, Southboro, MA, USA) run on a Dell computer. Subsequent analysis and graphing was performed using Pulsefit (HEKA), Mini analysis (Synaptosoft Inc., Decatur, GA, USA) and Igor Pro (Wavemetrics, Lake Oswego, OR, United States).

\section{ELECTRON MICROSCOPY}

N2, unc-18(b403), unc-18(md299), tom-1(ok285), unc-13(s69), syntaxin mosaics $\left(\mathrm{ACh}^{+} / \mathrm{GABA}^{-}\right)$, tom-1(ok285);unc-18(b403) and tom-1(ok285);unc-18(md299) young-adult hermaphrodites were prepared by HPF fixation, as previously described (Rostaing et al., 2004). Briefly, 15 animals were loaded into a specimen chamber filled with E. coli and immobilized by HPF at a temperature of $-180^{\circ} \mathrm{C}$ under high pressure in a Bal-Tec HPM010 and immediately moved to liquid nitrogen.

Freeze substitution was performed in a Reichart AFS machine (Leica, Oberkochen, Germany) as previously described (Weimer et al. 2006) using tannic acid (0.1\%) and $0.5 \%$ gluteraldehyde fixative introduced over 4 days and then stained with 2\% osmium. Fixed animals were then washed and embedded in Araldite 502 over a 48 -h period at $60^{\circ} \mathrm{C}$.

Serial sections were cut at a thickness of 40-50 nm, collected on formvar-covered carbon coated copper grids (EMS, FCF2010-Cu), and counterstained in $2 \%$ or $2.5 \%$ aqueous uranyl acetate for $4 \mathrm{~min}$, followed by Reynolds lead citrate for $2 \mathrm{~min}$. Images were obtained on a JEOL JEM-1220 transmission electron microscope (JEOL, Tokyo, Japan) operating at $80 \mathrm{kV}$. Micrographs were collected using a Gatan digital camera (Pleasanton, CA, USA).

Morphometric analysis was performed on serial sections containing ventral nerve cord neuromuscular synapses. The analysis was performed blind. Images were quantified using NIH Image software. An NMJ synapse was defined as a set of serial sections each containing a synaptic profile, identified on the basis of a presynaptic specialization adjacent to a muscle membrane and the presence of synaptic vesicles. Several morphometric measurements were obtained: synaptic vesicle density defined as the total number of synaptic vesicles per synaptic profile, the distance from each vesicle membrane perpendicular to the plasma membrane, and the distance to the proximal edge of the presynaptic specialization. Docked vesicles were defined as synaptic vesicles with $0 \mathrm{~nm}$ separation between vesicle and plasma membrane. Tethered vesicles were defined as synaptic vesicles between 1-25 $\mathrm{nm}$ from the plasma membrane. Both tethered and docked vesicles were graphed as percentage of total vesicle density per synaptic profile. 


\section{IMMUNO-ELECTRON MICROSCOPY}

Young-adult hermaphrodites for each strain were prepared by HPF as described above, fixed with $0.1 \%$ potassium permanganate for $72 \mathrm{~h}$ and embedded in Lowicryl for $60 \mathrm{~h}$ under UV light.

\section{UNC-18 antibody generation}

UNC-18 polyclonal antibodies were raised in rabbits by Cocalico Biologicals (Reamstown, PA, USA). Rabbits were inoculated with purified Histidine-tagged full-length UNC-18 protein expressed in E. Coli. Anti-UNC-18 antibodies were affinity purified by incubating whole rabbit sera with immobilized GST-tagged UNC-18 protein (expressed in E. Coli), then eluted with ActiSep Elution medium (Sterogene Bioseparations, Inc, Carlsbad, CA, USA). Eluted antibodies were desalted on G-25 Sepadex ${ }^{\mathrm{TM}}$ and concentrated before use.

\section{Post-embedding immunogold labeling}

Thin ( $50 \mathrm{~nm})$ sections from Lowicryl-embedded samples were collected as ribbons on formvar-coated nickel slot grids and immunogold labeled in a similar manner to that described previously (Rostaing et al., 2004). The UNC-18 primary antibody was used at a dilution of 1:500, and TOM-1 antibody was used at 1:300. Secondary anti-rabbit 10 or $15 \mathrm{~nm}$ gold bead-conjugated antibodies were diluted 1:100.

\section{WESTERN BLOT ANALYSIS}

Proteins were extracted from 50 larval stage four worms of each strain. Worm lysis and crude protein extraction were performed in $100 \mu$ l loading buffer: $0.35 \mathrm{M}$ Tris $\mathrm{pH}$ 6.8, 10\% SDS, 0.01\% Bromophenol blue, 35\% glycerol, $5 \% \beta$-mercaptoethanol containing 1:50 protease inhibitor cocktail plus $1 \mathrm{mM}$ EDTA $(\mathrm{pH} 8)$ by boiling for $10 \mathrm{~min}$ at $95^{\circ} \mathrm{C}$. A fast centrifugation to pellet worm fragments was performed before running $20 \mu \mathrm{L}$ of each sample through an $8 \%$ SDS-PAGE gel. Following protein transfer onto a PVDF membrane and preblocking with I-block, membranes were incubated in primary antibody (UNC-18 1:2000 or syntaxin 1:2,500 generated in rabbits) overnight at $4^{\circ} \mathrm{C}$, washed in TBS-T, and incubated in HRP-conjugated goat anti-rabbit for $1 \mathrm{~h}$, and washed again three times in TBS-T before adding luminescent solution for $5 \mathrm{~min}$ (see Pierce detection kit). Protein levels were normalized to anti-tubulin (1:500 primary) immunostaining of stripped membranes.

\section{RESULTS \\ UNC-18-DEPENDENT SYNAPTIC VESICLE TETHERING OCCURS IN THE ABSENCE OF DOCKING}

C. elegans unc-18 mutants exhibit defects in evoked and spontaneous synaptic transmission and a concomitant reduction in the size of the readily releasable synaptic vesicle pool (Weimer et al., 2003). These functional defects were previously attributed to a reduction in synaptic vesicle docking, a morphological step thought to be upstream of vesicle priming. This conclusion was based on comparison to priming defective unc-13 mutants, which exhibited a normal proportion of docked vesicles in EM sections derived from aldehyde fixed specimens (Richmond et al., 1999). However, recent implementation of HPF fixation of C. elegans unc-13 mutants revealed a severe synaptic vesicle docking defect, indicating that morphological docking is largely synonymous with vesicle priming under these fixation conditions (Weimer et al., 2006).

In order to resolve this apparent conflict in the data, and to better understand the morphological consequences of eliminating UNC-18, two loss of function alleles of C. elegans unc-18(b403 and $m d 299)$ were analyzed following HPF. Morphometric analyses of unc-18 mutant synaptic profiles by EM revealed a reduction in two vesicle pools; those docked at the plasma membrane (defined as $0 \mathrm{~nm}$ separation between vesicle and plasma membranes) and those tethered within one vesicle diameter ( $>0 \mathrm{~nm}$ up to $25 \mathrm{~nm}$ ) of the plasma membrane (Figure 1A). Specifically, the percentage of docked vesicles per synaptic profile in $u n c-18$ (b403) was reduced to $38 \%$ of wild-type $(p<0.0001$, Figures $1 \mathrm{~B}, \mathrm{D})$ and vesicle tethering was reduced to $43 \%(p<0.0001$, Figures 1B,E). Similar results were obtained for the second allele, unc-18( $m d 299)$ in which vesicle docking and tethering were reduced to $41 \%(p<0.0001)$ and $28 \%$ $(p<0.0001)$ of wild-type, respectively (data not shown).

In contrast to unc-18 mutants, unc-13(s69) mutants prepared by HPF (Figure 1C) exhibited reduced synaptic vesicle docking, (Figure 1D) (45\% of wild-type, $p<0.0001)$ as previously observed (Weimer et al., 2006; Hammarlund et al., 2007), but at the same time accumulated tethered synaptic vesicles (124\% of the wild-type, $p=0.0004$, Figure 1E). These results indicate that UNC-18 plays a role in vesicle tethering that is independent of vesicle priming.

\section{VESICLE DOCKING AND TETHERING ARE DEPENDENT ON UNC-18/ SYNTAXIN INTERACTIONS}

Munc18 is known to associate with the plasma membrane through syntaxin interactions (Rickman et al., 2007). To test whether $C$. elegans UNC-18 is similarly targeted to the plasma membrane in a syntaxin-dependent manner, we compared the levels of membrane-associated UNC-18 in synapses with and without syntaxin (UNC-64) by immunoEM analysis. Since C. elegans unc-64(js115) null mutants are larval lethal, we used a transgenic line in which viability was restored to $u n c-64(j s 115)$ mutants by expressing syntaxin (UNC-64) in cholinergic neurons (Hammarlund et al., 2007). Using these syntaxin mosaic animals we were able to compare the level of membrane-associated UNC-18 in cholinergic (syntaxin+) (Figure 2A) versus GABAergic (syntaxin-) (Figure 2B) neuromuscular junctions (NMJs) in the same worms. Whereas $67 \%$ of all synaptic immunogold bead labeling of UNC-18-specific antibodies was associated with the plasma membrane at cholinergic NMJs in mosaic animals (Figures 2A,C), GABAergic synapses were devoid of membrane-associated beads ( $0 \%$ of total beads) (Figures 2B,C). The percentage of plasma membrane-associated beads in cholinergic synapses of syntaxin mosaic animals was higher than that observed at wild-type synapses (38\%) (Figure 2C), which may reflect the overexpression of syntaxin in the mosaic transgenic line. Thus, UNC-18 association with the neuronal plasma membrane is dependent on the presence of syntaxin (UNC-64) in C. elegans.

Since UNC-18 requires syntaxin (UNC-64) for plasma membrane localization, we predicted that in the absence of syntaxin both docked and tethered vesicles would be impacted. To test this hypothesis, we prepared unc-64 (syntaxin) mosaic worms for morphological analysis following HPF fixation (Figures 3A,B). As previously published, (Hammarlund et al., 2007) we found that 


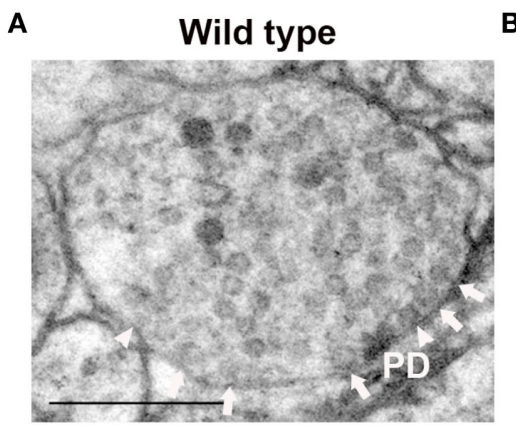

D

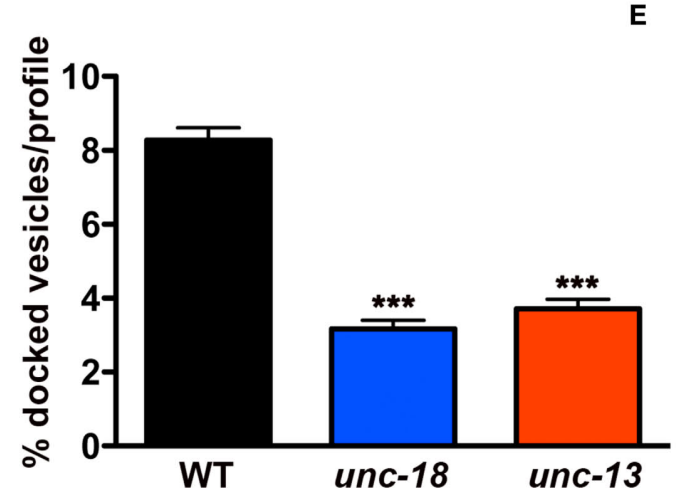

FIGURE 1 | unc-18 mutants exhibit defects in vesicle docking and tethering (A-C) Representative electron micrographs of NMJ profiles prepared by HPF fixation for morphometric analysis from (A) wild-type (B) unc-18(b403) mutant and (C) unc-13(s69) mutant. Arrowheads indicate plasma membrane docked synaptic vesicles, arrows indicate tethered synaptic vesicles $k 25 \mathrm{~nm}$ of the plasma membrane) and PD indicates the presynaptic density. Scale bar $200 \mathrm{~nm}$. unc-18

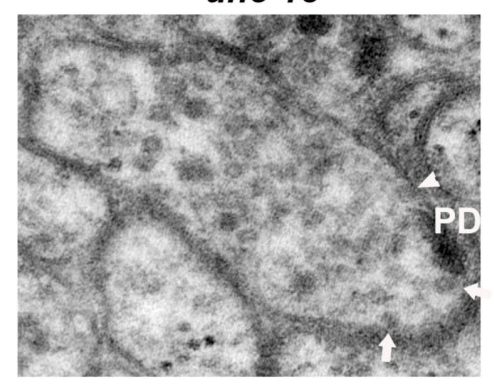

C

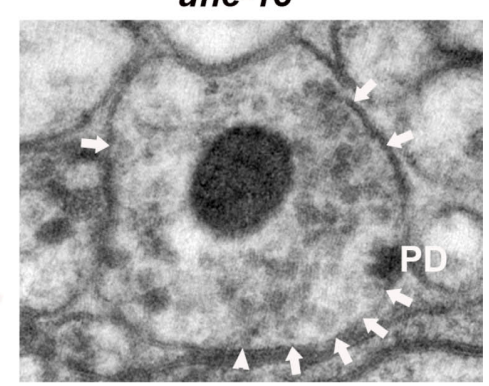

E

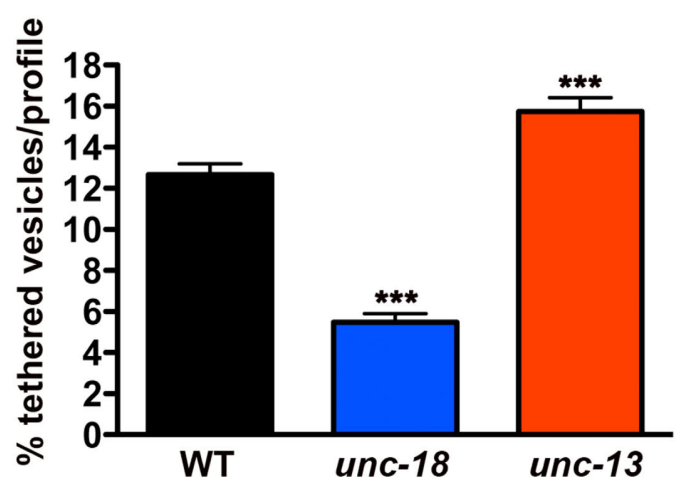

(D) Average docked and (E) tethered synaptic vesicles expressed as a percentage of total vesicles per synaptic profile for wild-type ( $n=178$ synaptic profiles, vesicle density $20.2 \pm 0.6$ ), unc-18(b403) ( $n=60$ synaptic profiles, vesicle density $35.2 \pm 1.4$ ) and unc-13(s69) ( $n=101$ synaptic profiles, vesicle density $24.4 \pm 0.8$ ). Statistical values represent mean \pm SEM in this and subsequent figures. ${ }^{* *} p<0.001$ relative to wild-type.

GABAergic synapses lacking syntaxin exhibited a large reduction in vesicle docking $(66 \%)$ compared to syntaxin-expressing cholinergic neurons in the same worms $(p<0.0001)$ (Figure 3C). In addition, we found tethered vesicles were also reduced by $58 \%$ in the GABAergic synapses when compared to cholinergic synapses $(p<0.0001)$ (Figure 3D). These results indicate that both tethering and docking of synaptic vesicles are UNC-18 and syntaxin dependent.

Vertebrate Munc18-1 is known to interact with closed and open conformations of syntaxin; these binding modes implicated in different Munc18-1-dependent stages of vesicle exocytosis (Gulyas-Kovacs et al., 2007; Rickman et al., 2007; Gerber et al., 2008). To test whether the closed synaxin/UNC-18 complex is required for either the tethering or docking of synaptic vesicles at $C$. elegans synapses, we examined syntaxin null mutants rescued with mutated syntaxin (UNC-64 L166A/E167A), predicted to render syntaxin constitutively open (Dulubova et al., 1999; Richmond et al., 2001). Synapses expressing open syntaxin (Figure 4A) exhibited an approximately twofold higher ratio of docked vesicles $(210 \%)(p<0.0001)$ when compared to wild-type (Figure 4B), an increase that is likely due to overexpression of open syntaxin based on Western blot analysis. In contrast tethered vesicles were $26 \%$ lower than wild-type $(p=0.0009)$ (Figure 4C), indicating that disruption of the UNC-18/closed syntaxin binding mode negatively impacts the tethering step, whereas UNC-18/open syntaxin promotes vesicle docking. Thus, the involvement of UNC-18 in tethering and docking of synaptic vesicles depends on differential molecular interactions with the integral plasma membrane SNARE protein, syntaxin.

\section{TOM-1 INHIBITS UNC-18-DEPENDENT VESICLE DOCKING AND TETHERING}

Vertebrate tomosyn has been shown to compete with Munc18-1 for syntaxin binding, both in vitro (Fujita et al., 1998) and in vivo (Gladycheva et al., 2007). Consistent with these observations, we found that the level of TOM-1 plasma membrane-association assessed by immunoEM was reduced by $67 \%$ in the GABAegic (syntaxin-) synapses when compared to cholinergic (syntaxin+) synapses in the syntaxin mosaic worms and to a similar extent when compared to wild-type synapses (Figures 2D-F). To determine whether TOM-1 and UNC-18 compete for binding to membranebound syntaxin, we next examined membrane-associated TOM-1 and UNC-18 in $u n c-18$ and tom- 1 mutants by immunoEM. Plasma membrane-associated anti-TOM-1 labeling was enhanced by $33 \%$ in $u n c-18$ mutants (Figures 5A,C), when compared to wild-type synapses. Conversely, the proportion of syntaxin-dependent plasma membrane localized UNC-18 increased by $108 \%$ in the tom-1 mutant background, when compared to wild-type synapses (Figures 5B,D). 

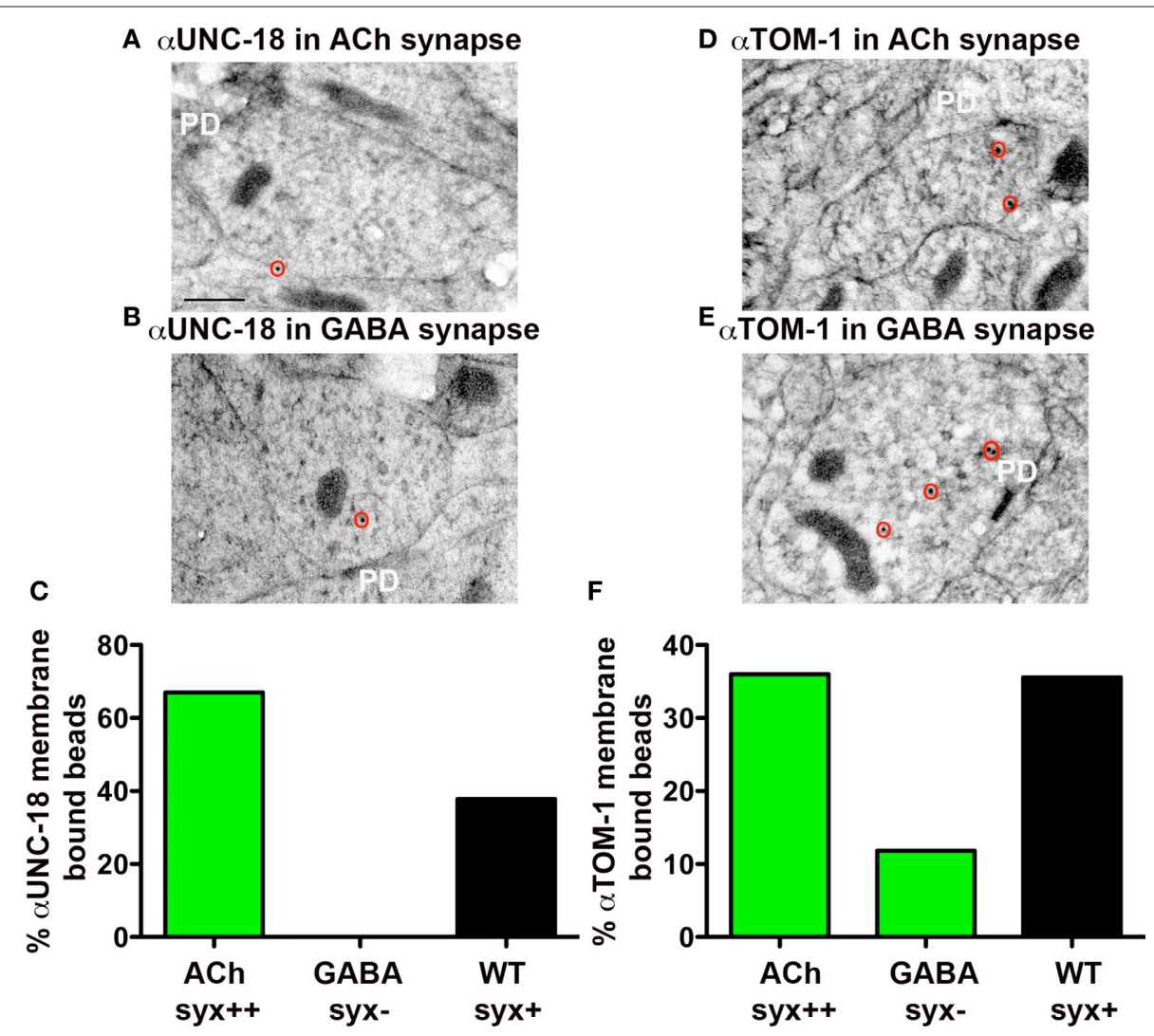

FIGURE 2 | Plasma membrane-association of UNC-18 and TOM-1 is dependent on syntaxin. Representative micrographs prepared for immunoEM, showing immunogold labeling of UNC-18 (A,B) and TOM-1 (D,E) in cholinergic syntaxin-expressing NMJs (upper micrographs) and GABAergic syntaxin null NMJs (lower micrographs) from syntaxin mosaic worms (strain EG3278). Immunogold particles are circled. PD indicates the presynaptic density. Scale bar $200 \mathrm{~nm}$. (C,F) The percentage of total synaptic beads that are associated with the plasma membrane $(>30 \mathrm{~nm}$ ) in cholinergic (syntaxin++) and

GABAergic (syntaxin-) synapses of syntaxin mosaic worms (green bars, ) compared to wild-type synapses (black bars) using antibodies recognizing UNC-18 (C) and TOM-1 (F). Bead counts were as follows: Anti-UNC-18 (wild-type synapses: 41 synaptic profiles, 56 beads, syntaxin mosaics: 8 cholinergic synaptic profiles, 12 beads, 7 GABAergic synaptic profiles, 7 beads). Anti-TOM-1 (wild-type synapses: 104 synaptic profiles, 154 beads, syntaxin mosaics: 28 cholinergic synaptic profiles, 29 beads, 8 GABAergic synaptic profiles, 16 beads).

Based on competition between UNC-18 and TOM-1 for syntaxin binding, we hypothesized that loss of TOM-1 would enhance UNC-18/syntaxin interactions and therefore increase the number of tethered as well as docked synaptic vesicles. Analysis of tom-1 mutants confirmed this prediction; the proportion of docked vesicles per profile increasing by $93 \%$ in tom-1(ok285) when compared to wild-type synapses $(p<0.0001$, Figures 6A,B), as previously demonstrated (Gracheva et al., 2006), and in addition vesicle tethering increased by $71 \%(p<0.0001$, Figures $6 \mathrm{~A}, \mathrm{C})$, indicating that TOM-1 negatively regulates both UNC-18-dependent vesicletargeting steps. Similar results were obtained with a second allele of tom-1 (nu468) in which docking was increased by $84 \%(p<0.0001)$ and tethering by $53 \%(p<0.0001$, data not shown).

\section{TOM-1 PREFERENTIALLY RESCUES SYNAPTIC VESICLE DOCKING IN unc-18 MUTANTS}

Given the opposing roles of TOM-1 and UNC-18 in vesicle targeting, we next addressed whether removal of TOM-1 rescued either of the vesicle targeting defects of unc-18 mutants. In tom-1;unc-18 double mutants there was minimal (although significant, $p=0.02$ ) improvement in the ratio of tethered vesicles; tom-1;unc-18 doubles (54\% of wild-type) compared to $u n c-18$ alone ( $43 \%$ of wild-type,) (Figures 7A,C) whereas vesicle docking was substantially restored in tom-1;unc-18 doubles ( $75 \%$ of wild-type) compared to $u n c-18$ alone $(38 \%$ of wild-type) $(p<0.0001$, Figures 7A,B).

In previous HPF studies, we have observed a strong correlation between the size of the morphologically docked vesicle pool and the level of priming (Gracheva et al., 2006). Therefore, we predicted that tom-1;unc-18 double mutant synapses would show some functional recovery relative to $u n c-18$, reflecting the increase in vesicle docking. Electrophysiological analysis of tom-1;unc-18 double mutants confirmed that the increase in docked vesicles was associated with improved release when compared to $u n c-18$ single mutants ( $p=0.0031$, Figures 7D,E). Specifically, the average evoked response of tom-1;unc-18 double mutants was $38 \%$ of wild-type, whereas unc-18 single mutants was only $13.2 \%$ (Figure 7E). To determine whether the improvement in exocytosis reflected enhanced priming in tom-1;unc-18 double mutants we measured synaptic responses to hyperosmotic saline (Figures 7D,F). As expected tom-1; unc-18 
A

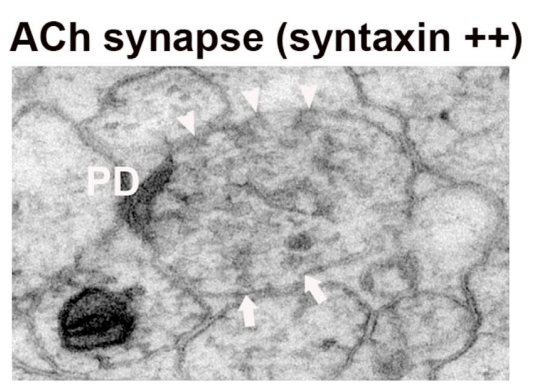

B

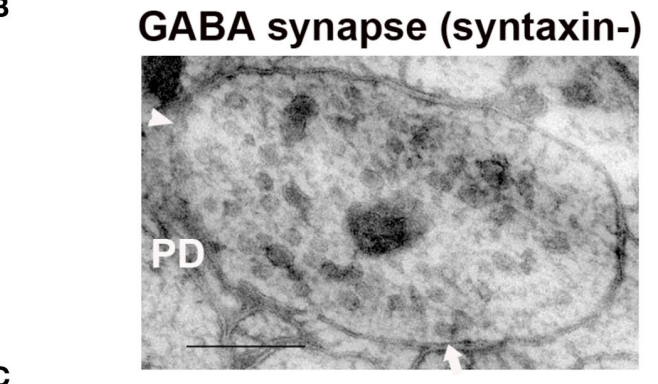

C

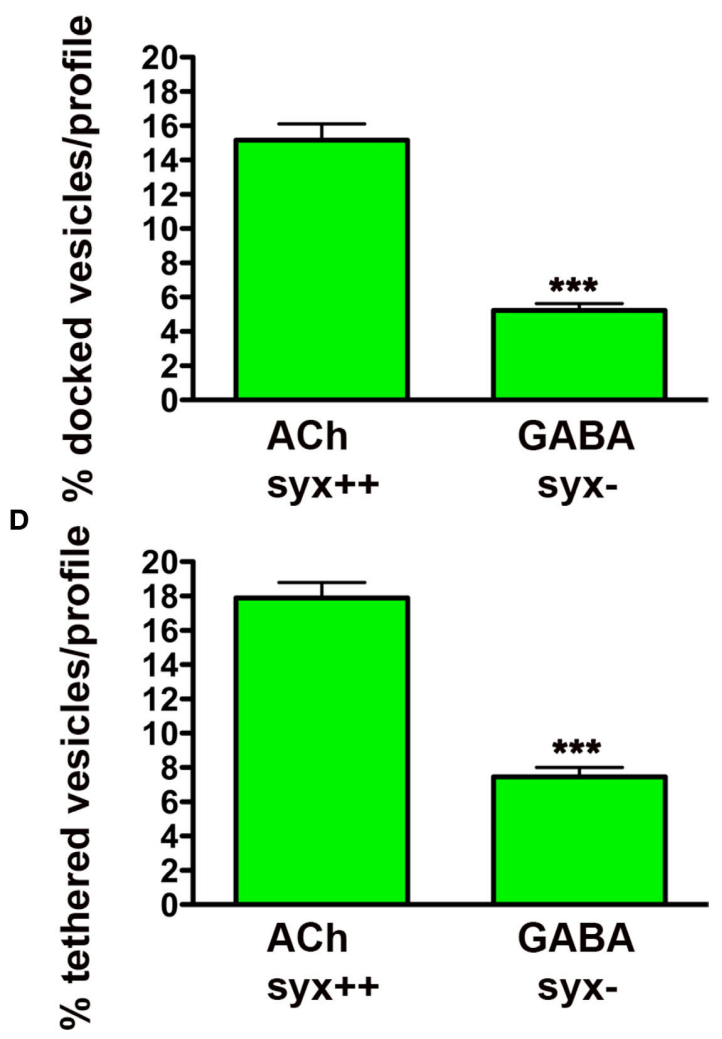

FIGURE 3 | Synaptic vesicle tethering and docking are syntaxin-dependent. Representative electron micrographs of NMJ profiles of syntaxin mosaic animals (strain EG3278) which have cholinergic syntaxin-expressing synapses (A) and GABAergic syntaxin null synapses (B). Arrowheads indicate plasma membrane docked synaptic vesicles, arrows indicate tethered synaptic vesicles $(<25 \mathrm{~nm}$ of the plasma membrane), PD presynaptic density, scale bar $200 \mathrm{~nm}$. Average docked (C) and tethered (D) synaptic vesicles expressed as a percentage of total vesicles per synaptic profile for cholinergic syntaxin-expressing synapses (syx++, $n=24$ synaptic profiles, vesicle density $25 \pm 1.0$ ) and GABAergic syntaxin null synapses (syx-, $n=35$ synaptic profiles, vesicle density $37.1 \pm 1.6)$. ${ }^{* *} p<0.001$ GABAergic synapses relative to cholinergic synapses.

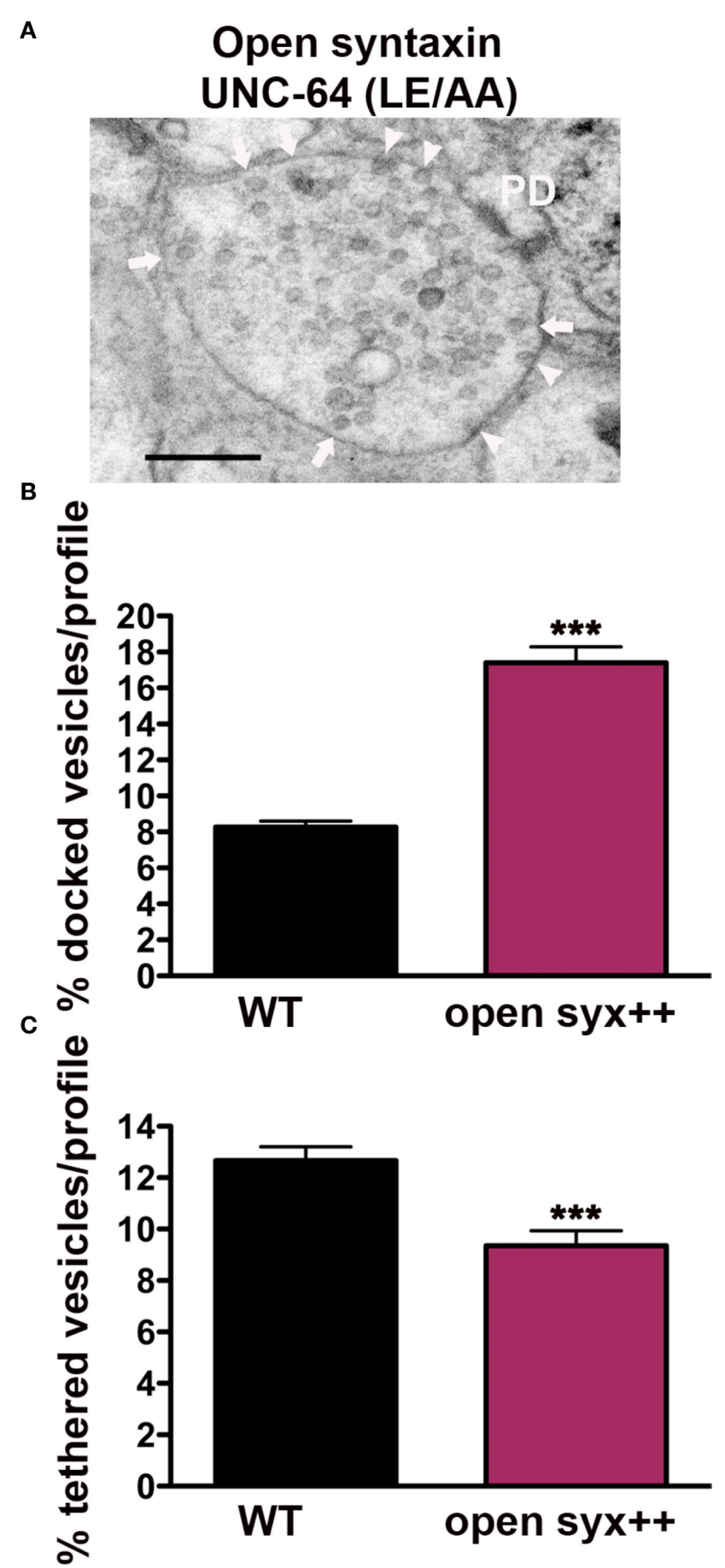

FIGURE 4 | Synaptic vesicle tethering and docking are differentially regulated by syntaxin open state conformation. (A) Representative electron micrograph of an NMJ profile from syntaxin null mutants rescued with open syntaxin (LE166/167AA) (strain EG1985). Arrowheads indicate plasma membrane docked synaptic vesicles, arrows indicate tethered synaptic vesicles ( $<25 \mathrm{~nm}$ of the plasma membrane), PD presynaptic density, scale bar $200 \mathrm{~nm}$. Average docked (B) and tethered (C) synaptic vesicles expressed as a percentage of total vesicles per synaptic profile. Open syntaxin ( $n=59$ synaptic profiles, vesicle density $29.8 \pm 1.2$ ), wild-type $(n=178$ synaptic profiles, vesicle density $20.2 \pm 0.6) .{ }^{* *} p<0.001$ relative to wild-type. 


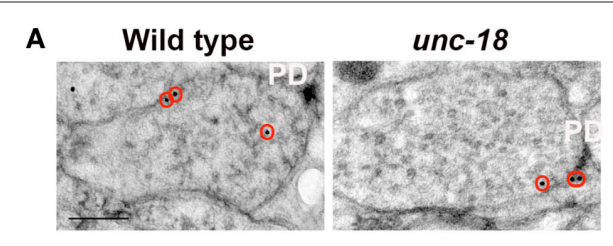

c

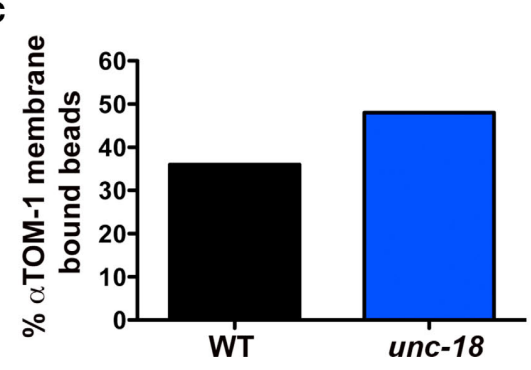

FIGURE 5 | UNC-18 andTOM-1 compete for plasma membrane-association. Representative micrographs showing immunogold labeling of TOM-1 in wild-type and unc-18(md299) mutant synapses (A) and UNC-18 labeling in NMJs of wild-type and tom-1(ok285) mutants (B). Immunogold particles are circled. PD indicates the presynaptic density. Scale bar $200 \mathrm{~nm}$. (C, D) The percentage of total synaptic beads that are associated with the plasma

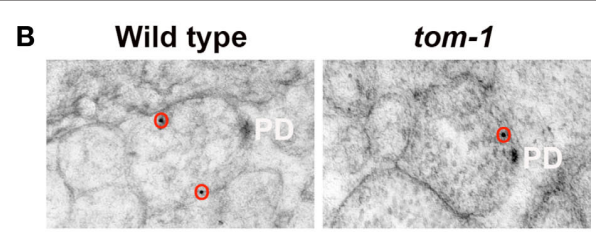

D

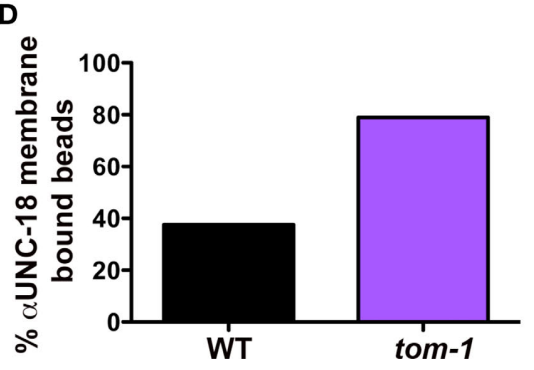

membrane (>30 nm) in TOM-1 labeled unc-18 mutants versus wild-type synaptic profiles (C) and UNC-18 labeling in tom-1 mutant synapses compared to wild-type (D). Bead counts were as follows: Anti-UNC-18 (wild-type: 41 synaptic profiles, 56 beads, tom-1 (ok285): 31 synaptic profiles, 38 beads). Anti-TOM-1 (wild-type: 104 synaptic profiles, 154 beads, unc-18(md299): 57 synaptic profiles, 93 beads) double mutants had increased hyperosmotic responses $(62.5 \%$ of wild-type) relative to $u n c-18$ mutants alone (23\% of wild-type) $(p=0.01$, Figure 7F).

Previous studies have demonstrated that, unc-18 mutants have a $\sim 50 \%$ reduction in syntaxin levels, attributed to loss of an UNC-18 chaperone function (Weimer et al., 2003; McEwen and Kaplan, 2008). Therefore, to test whether the improvement in vesicle priming in tom-1;unc-18 double mutants was the result of increased syntaxin levels we performed Western blot and densitometry analysis. tom-1;unc-18 double mutants exhibited a similar reduction in syntaxin levels (43\%) as $u n c-18$ single mutants (61\%) (Figures 8A,B), corresponding to the complete loss of UNC-18 protein (Figures 8A,C). Thus, the increased priming in tom-1;unc-18 double mutants compared to $u n c-18$ single mutants appears to reflect the removal of inhibitory TOM-1 binding to the remaining syntaxin, rather than partial recovery of syntaxin levels.

\section{DISCUSSION}

The results of this study provide evidence for two morphologically and, molecularly distinct synaptic vesicle targeting steps; a tethering step involving UNC-18/ closed syntaxin interactions, and a membrane docking step requiring both UNC-18 and open syntaxin that renders vesicles fusion competent. We further demonstrate that both tethering and docking of synaptic vesicles are negatively regulated by TOM-1, which competes with UNC-18 for syntaxin binding.

In our previous EM analysis of unc-18 mutant synapses, using conventional aldehyde fixation, we reported a reduction in docked vesicles, defined as vesicles within one vesicle diameter of the plasma membrane (Weimer et al., 2003). Our present ability, to divide this docked pool into two molecularly distinct UNC-18-dependent targeting steps is the result of applying rapid freeze fixation techniques which immobilize synaptic components on a millisecond time-scale avoiding artifacts associated with chemical fixation (Siksou et al., 2007). The summed loss of both tethered and docked vesicles in unc-18 mutants accounts for the previously observed docking defect (Weimer et al., 2003). Furthermore, the observation that unc-13 mutants prepared by HPF fixation accumulate tethered vesicles while losing morphologically docked vesicles, explains why we did not previously detect a docking defect in $u n c-13$ mutants following chemical fixation, as the sum of these two pools approaches that of wild-type synapses (Richmond et al., 1999). This latter result is consistent with the analysis of mouse Munc13-1/2 double knockout synapses following HPF fixation, in which vesicle docking was also found to be reduced while tethered vesicles accumulated (Siksou et al., 2009), negating the previous report of normal vesicle docking in Munc13-1 mutants following chemical fixation (Varoqueaux et al., 2002). Our present results in C. elegans suggest that the majority of tethered vesicles observed in Munc13-1/2 mutants are likely dependent on Munc18/closed syntaxin interactions, a prediction that will require ultrastructural analysis of mouse Munc18-1 and syntaxin-1 mutant synapses following HPF fixation.

In both the present study and previous studies characterizing unc-18 mutants a 50\% decrease in syntaxin levels was observed (Weimer et al., 2003; McEwen and Kaplan, 2008). Although the level of syntaxin at ventral synapses of unc-18 mutants was not directly measured, an estimate based on reduced overall syntaxin levels and $\%$ accumulation of syntaxin in cell-bodies suggested 35\% syntaxin remains at synapses (McEwen and Kaplan, 2008) with minimal impact on the percentage of membrane-bound syntaxin, based on immunoEM (Weimer et al., 2003). While we can't address the possible effect of reduced syntaxin on vesicle tethering, we do not think this accounts for the severe defects in docked, fusion competent vesicles observed in unc-18 mutants for the following 
A

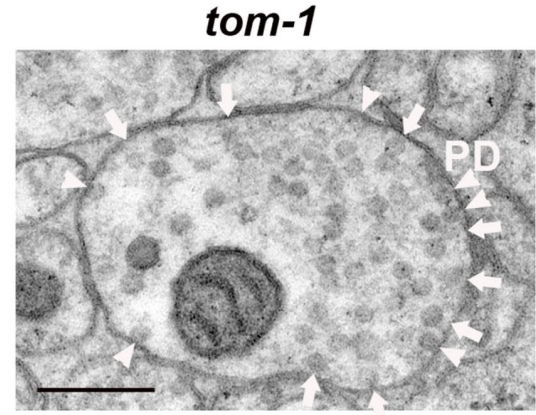

B

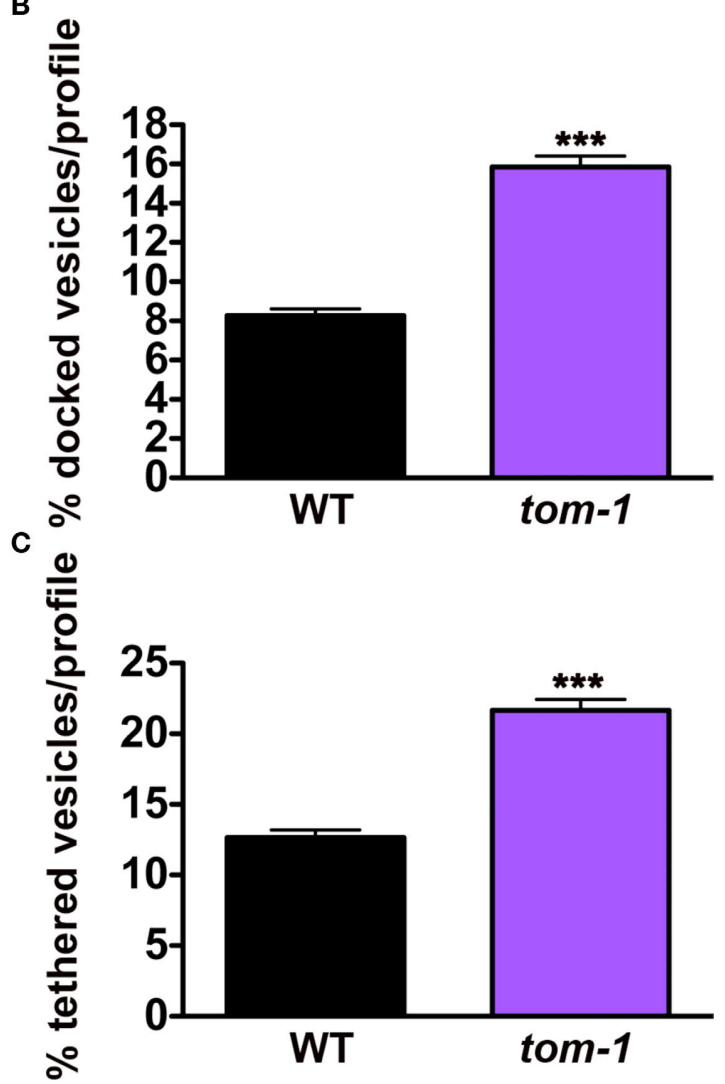

FIGURE 6 |TOM-1 negatively regulates synaptic vesicle docking and tethering. (A) Representative electron micrograph of an NMJ profile from a tom-1 (ok285) mutant. Arrowheads indicate plasma membrane docked synaptic vesicles, arrows indicate tethered synaptic vesicles $<25 \mathrm{~nm}$ of the plasma membrane), PD presynaptic density, scale bar $200 \mathrm{~nm}$. Average docked (B) and tethered (C) synaptic vesicles expressed as a percentage of total vesicles per synaptic profile. tom-1 (ok285) ( $n=115$ synaptic profiles, vesicle density $22.1 \pm 0.9$ ), wild-type ( $n=178$ synaptic profiles, vesicle density $20.2 \pm 0.6) .{ }^{* * *} p<0.0001$ relative to wild-type.

reasons. First, we have previously demonstrated that in unc-64 heterozygotes, syntaxin levels are reduced to $30 \%$ of the wild-type (Weimer et al., 2003) and yet evoked release amplitude is unaffected, indicating that this level of syntaxin is sufficient to support normal levels of docked and primed vesicles. In contrast unc-18 mutants with similar reductions in synaptic syntaxin levels have severely impaired levels of docked, primed vesicles. Second, overexpression of syntaxin in $u n c-18$ mutants does not rescue evoked release, as measured by evoked response amplitudes, thus increasing syntaxin levels does not bypass the requirement for UNC-18 in priming (Weimer et al., 2003). Third, the fact that unc18;tom-1 mutants exhibit significant rescue of docked, primed vesicles despite having a similar reduction in syntaxin to unc-18 single mutants suggests that there is sufficient syntaxin at the synapse in the absence of UNC-18 to support more docking. Based on these observations we propose that $u n c-18$ mutant defects in vesicle tethering and docking are not simply due to reduced syntaxin levels, but rather reflect a specific requirement for UNC-18 in these two processes.

How the UNC-18/closed syntaxin interaction mediates the membrane tethering of vesicles is presently unknown and could involve either (or both) of the following mechanisms: (1) UNC-18/ closed syntaxin complexes may actively promote the tethering of synaptic vesicles at the plasma membrane via interactions with an unidentified vesicle tethering factor or (2) the presence of UNC-18/ closed syntaxin dimers may interfere with the completion of SNARE complex-dependent morphological docking, resulting in vesicles tethered by partially or fully assembled SNARE complexes spatially separated from the plasma membrane by UNC-18/closed syntaxin dimers. In support of the former mechanism, synaptotagmin has recently been proposed to act as a vesicle tethering factor in the Munc18-dependent docking of dense core vesicles (de Wit et al., 2009). Specifically, vesicle docking in Munc18-1 mutant chromaffin cells can be rescued by manipulations that stabilize SNAP-25/ syntaxin dimers (de Wit et al., 2009), a process normally promoted by Munc18-1 (Burkhardt et al., 2008; Rodkey et al., 2008). Evidence suggests that stabilized SNAP-25/syntaxin dimers can then act as acceptor complexes for vesicle-associated synaptotagmin-1, resulting in the restoration of vesicle docking in the absence of Munc18-1 (de Wit et al., 2009). Since the Munc18-1 mutant chromaffin cells in these studies were chemically fixed, we postulate that the docking defects observed could actually represent loss of both tethered and docked vesicles. Evidence for the existence of tethered secretory granules has recently been reported in pancreatic cell lines using TIRFM (Karatekin et al., 2008). Tracking vesicle trajectories revealed secretory granules tethered $\sim 20 \mathrm{~nm}$ from the plasma membrane for often 10s of seconds, prior to docking and subsequently fusing with the plasma membrane in a calcium-dependent manner (Karatekin et al., 2008). These data indicate that tethered vesicles represent a prefusogenic state. A similar molecular mechanism could be at play in the tethering of synaptic vesicles at $C$. elegans synapses, since we know this tethering process is UNC-18 and syntaxin-dependent and acts upstream of the priming process required for fusion (based on the accumulation of tethered vesicles in priming defective $u n c-13$ mutants). If this tethering process occurs at C. elegans synapses, we would expect to see tethering defects in C. elegans SNAP-25(RIC-4) and synaptotagmin-1(SNT-1) mutants, as loss of these proteins in chromaffin cells exhibit docking defects similar to Munc18-1 null mutants (de Wit et al., 2009). Unfortunately, C. elegans ric-4 null mutants are embryonic lethal precluding EM analysis and snt-1 mutants exhibit profound reductions in vesicle density due to recycling defects (Jorgensen et al., 1995), thus the tethered and docked vesicle pools in these mutants can not be directly compared to those of unc-18 mutants. Similarly, interpretation of the docking defects observed at Drosophila synaptotagmin mutant synapses as well as dissociated hippocampal cultures from 
A

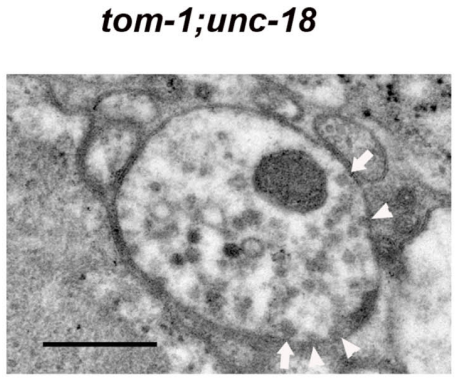

B

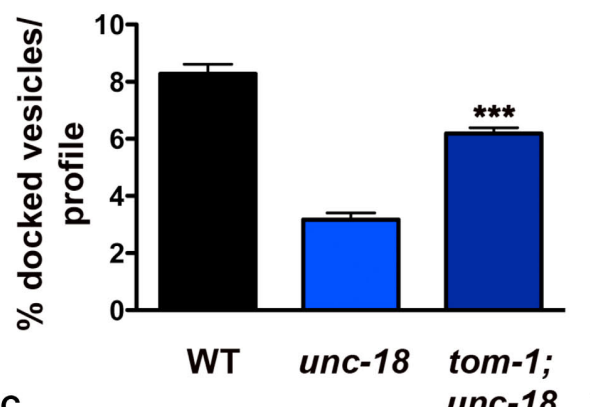

$c$

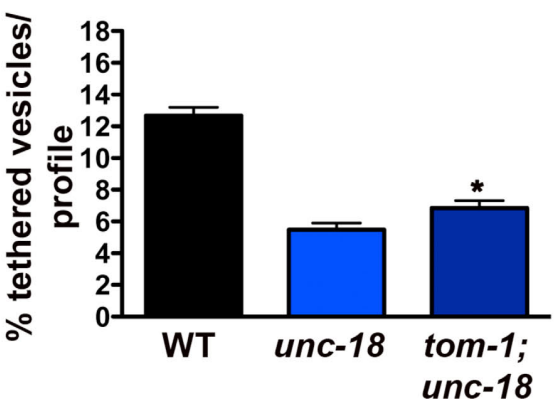

D

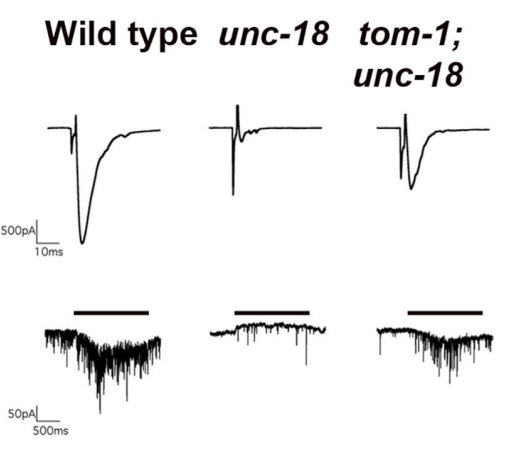

$\mathbf{E}$

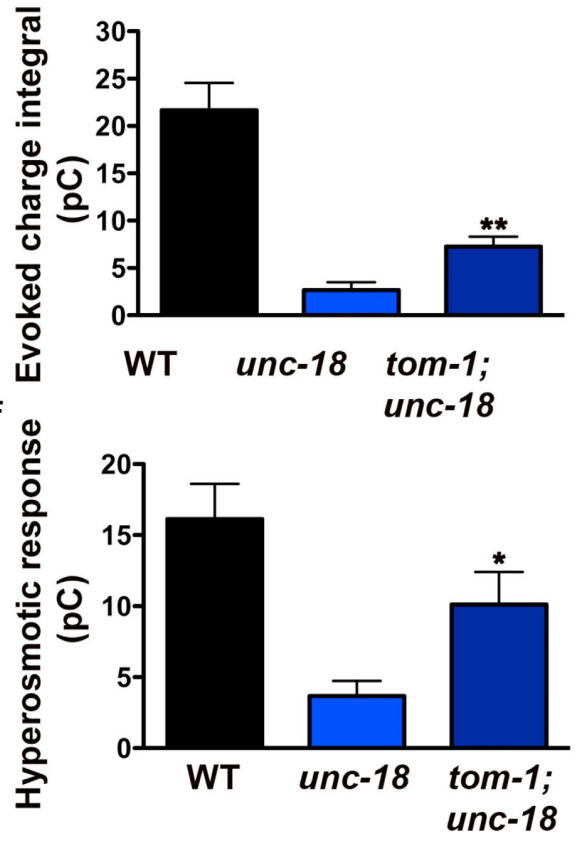

FIGURE 7 | tom-1 mutants partially rescue synaptic function in unc-18 mutants due to an increase in the docked, fusion competent synaptic vesicle pool. (A) Representative electron micrograph of an NMJ profile from a tom-1 (ok285); unc-18(b403) double mutant. Arrowheads indicate plasma membrane docked synaptic vesicles, arrows indicate tethered synaptic vesicles ( $<25 \mathrm{~nm}$ of the plasma membrane), PD presynaptic density, scale bar $300 \mathrm{~nm}$. Average docked $(\mathbf{B})$ and tethered $\mathbf{( C )}$ synaptic vesicles expressed as a percentage of total vesicles per synaptic profile for wild-type ( $n=178$ synaptic profiles, vesicle density $20.2 \pm 0.6)$, unc-18(b403) ( $n=60$ synaptic profiles, vesicle density $35.2 \pm 1.4$ ) and tom-1 (ok285); unc-18(b403) ( $n=68$ synaptic profiles, vesicle density $33.1 \pm 1.2$ ). (D) Representative evoked responses (upper traces) and hyperosmotic responses (lower traces) in voltage-clamped body wall muscles of dissected wild-type, unc-18 and tom-1;unc-18 strains. Muscle holding potential -100 mv, evoked stimulation 2 ms depolarization of ventral nerve cord, hyperosmotic application indicated by horizontal bar above traces. Average evoked charge integral (E) and hyperosmotic response charge integral (F) for wild-type, unc-18 and tom-1; unc-18. ${ }^{*} p<0.05,{ }^{* *} p<0.01,{ }^{* * *} p<0.001$ tom-1 (ok285); unc-18(b403) double mutants relative to unc-18(b403) alone. mouse synaptotagmin-1 mutants is complicated by corresponding endocytic defects resulting in reduced vesicle number (Reist et al., 1998; Liu et al., 2009). Therefore, at this point we cannot with any certainty, conclude that $C$. elegans synaptic vesicles are either tethered or docked via UNC-18 mediated synaptotagmin/SNARE interactions, only that UNC-18 and syntaxin are involved in both vesicle targeting steps.

Ultrastructural evidence linking UNC-18 and syntaxin to either synaptic vesicle tethering or docking in other systems has produced mixed results. Analysis of vesicle docking in Drosophila UNC-18 (Rop) null mutant synapses is unavailable (Harrison et al., 1994), whereas syntaxin null mutant flies are reported to have increased numbers of docked vesicles at chemically fixed synapses (Broadie et al., 1995). This latter result conflicts with the profound tethering and docking defects that we observe at C. elegans syntaxin mutant synapses following HPF fixation (Hammarlund et al., 2007), and may reflect perdurance of maternal syntaxin in the small number of NMJs that form in embryonically lethal Drosophila null mutants (Featherstone and Broadie, 2002). However, there is also a lack of evidence for the involvement of syntaxin in synaptic vesicle docking at squid (O'Connor et al., 1997) and mammalian synapses (de Wit et al., 2006). In contrast, up and down regulation of Munc181-levels in hippocampal autapses results in modest bidirectional changes in synaptic vesicle docking that correspond to changes in the readily releasable vesicle pool size (Toonen et al., 2006). This result is consistent with observed synaptic vesicle docking defects in the neocortex of late but not in early stage embryos or cultured cortical neurons from Munc18-1 null mutants (Verhage et al., 


\section{A

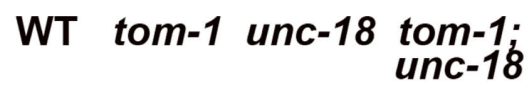

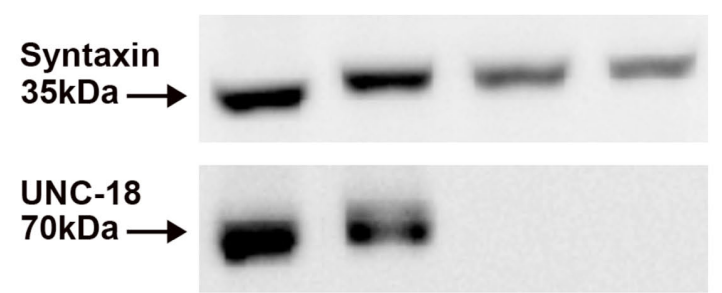

B
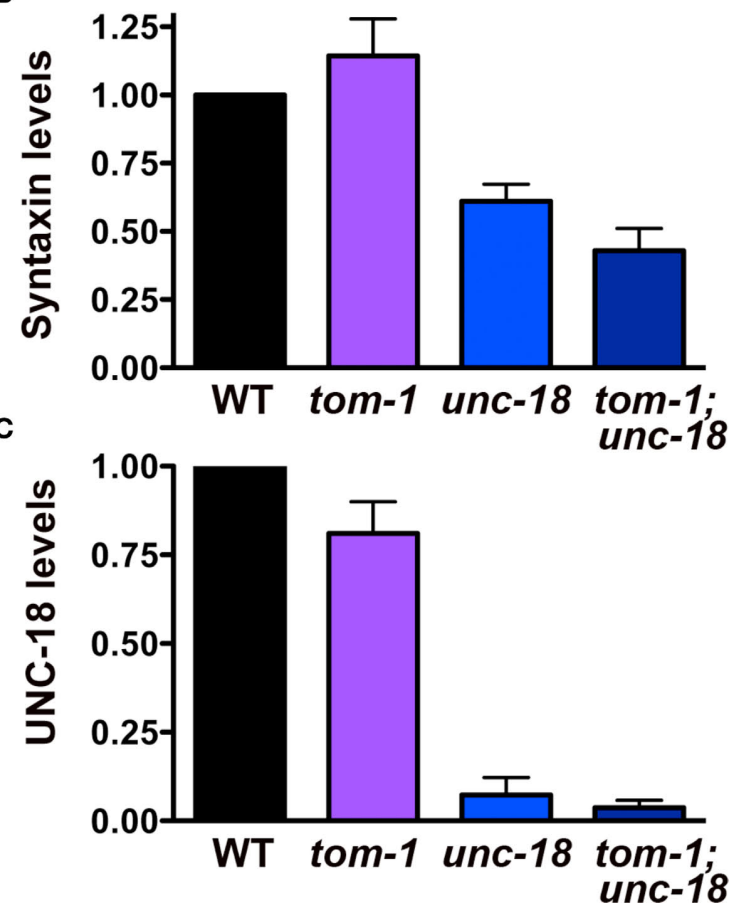

FIGURE 8 | tom-1 mutants do not affect syntaxin levels in either wild-type or unc-18 mutant backgrounds. (A) Representative Western blots derived from 50 lysed larval stage four worms probed with antibodies against syntaxin (upper blot) or UNC-18 (lower blot). Lanes: (1) wild-type (2) tom-1 (ok285) (3) unc-18(md299) (4) tom-1 (ok285);unc-18(md299). (B) Averaged syntaxin levels normalized to anti-tubulin immunostaining of stripped membranes $(n=3$ experiments). (C) Averaged UNC-18 levels normalized to anti-tubulin immunostaining of stripped membranes ( $n=3$ experiments).

2000; Bouwman et al., 2004; Gerber et al., 2008). Why there is so much variability across species in the extent to which synaptic vesicle tethering and docking are impacted by loss of Munc-18 and syntaxin remains to be determined and likely reflects differences in experimental methods such as genetic deletion versus toxin cleavage, developmental stage at which synapses were examined, in vivo versus cell culture conditions, as well as chemical versus rapid freeze fixation.

The roles of Munc18 and syntaxin in dense core vesicle targeting have been investigated by both TIRFM and conventional EM. TIRFM real time imaging reveals changes in both delivery and retention time of vesicles at the plasma membrane in Munc18 mutant chromaffin cells, as well as reduced vesicle jitter, a readout of tethering forces (Toonen et al., 2006). The reduction of both vesicle delivery and membrane retention observed by TIRFM are expected to correlate with a reduction in docked vesicles at the ultrastructural level. This is indeed the case for Munc18-1 null mutant chromaffin cells, which exhibit a profound reduction in docked dense core vesicles in electron micrographs prepared by chemical fixation (Voets et al., 2001). In contrast, TIRFM following disruption of syntaxin function in chromaffin cells revealed no alteration in vesicle delivery or plasma membrane retention, only reduced tethering forces, thus the level of docking in these cells would be predicted to be normal. However, disruption of syntaxin in chromaffin cells has dramatic vesicle docking defects (de Wit et al., 2006). Therefore, attempting to correlate the residency time of vesicles at the plasma membrane of chromaffin cell dense core vesicles observed by TIRFM analysis with changes in vesicle docking based on classical EM, presents some inconsistencies. It also remains to be seen whether the vesicles that appear docked in chromaffin cells following chemical fixation can be further subdivided into tethered $(25 \mathrm{~nm})$ and primed $(0 \mathrm{~nm})$ states if these cells are prepared by HPF fixation, and whether, as in the case of C. elegans unc-18 and unc-64(syntaxin mutants), chromaffin cells lacking Munc18-1 or syntaxin function, exhibit defects in both pools.

What then is the functional role of UNC-18/closed syntaxindependent vesicle tethering? Analysis of mammalian synapses expressing constitutively open syntaxin suggests that loss of UNC-18/closed syntaxin dimers reduces the size of the primed vesicle pool while at the same time enhancing release rate (Gerber et al., 2008). The loss of primed vesicles in these synapses correlated with reduced levels of open syntaxin and Munc18 at the synapse, trafficking and stabilization of both proteins requiring Munc18/ closed syntaxin chaperone function. However, the basis for the increased release probability in these open syntaxin mutants is unknown. Our demonstration that vesicle tethering is dependent on UNC-18/closed syntaxin interactions provides a possible explanation for this, if UNC-18/closed syntaxin interactions interfere with progression to the fully primed state, a model proposed by Gerber et al. (2008).

While Munc18/closed syntaxin dimers reduce release probability, this result does not exclude the possibility that Munc18/closed syntaxin-dependent tethering also plays a permissive role in vesicle maturation beyond the chaperone function. Indeed, Munc18-1 defective in closed syntaxin binding, exhibits dense core granule docking and priming defects when overexpressed in Munc18-1 null chromaffin cells, despite restoration of greater than wild-type levels of membrane-associated syntaxin and Munc18 (Gulyas-Kovacs et al., 2007). Thus vesicle tethering may play a positive role upstream of vesicle priming. The extent of the synaptic vesicle docking defect observed in the absence of Munc18-1/closed syntaxin interactions is less than that exhibited by Munc18 null chromaffin cells, suggesting that docking, assessed by chemical fixation in these cells, likely represents two distinct pools of granules. We postulate that the large fraction of docked granules dependent on Munc18/closed syntaxin binding may be the dense core vesicle correlate of the synaptic vesicle tethered pool observed at C. elegans synapses following HPF fixation. Interestingly, while Munc18 mutants defective in closed syntaxin dimerization rescue only $23 \%$ of granule docking in Munc18 null chromaffin cells, $60 \%$ of release is rescued. These data suggest that while Munc18/closed syntaxin-dependent 
docking is necessary for the development of a full complement of primed vesicles, docked granules in the absence of Munc18/ closed syntaxin have a higher release probability (Gulyas-Kovacs et al., 2007). This result is consistent with the observed increase in synaptic vesicle release probability of open syntaxin mutants at mammalian synapses (Gerber et al., 2008) and strengthens the argument that while playing permissive roles in protein stabilization and vesicle tethering, Munc18/closed syntaxin dimerization also interferes with progression to the fully primed vesicle state.

In contrast to UNC-18, TOM-1 appears to negatively regulate both the tethering and docking of synaptic vesicles at $C$. elegans synapses (Gracheva et al., 2006). Our observations suggest that the accumulation of tethered vesicles in tom-1 mutants likely reflects enhanced UNC-18/closed syntaxin dimerization in the absence of competition for open syntaxin binding by TOM-1. This conclusion is supported by our immunoEM data showing that membrane localization of both UNC-18 and TOM-1 is syntaxin-dependent and that the ratio of plasma membrane-associated UNC-18 is increased in tom-1 mutants. These results complement in situ observations obtained by FRET analysis in HEK-293 and chromaffin cells, in which tomosyn was shown to compete with Munc18 for binding to plasma membrane-associated syntaxin (Gladycheva et al., 2007), as well as the original in vitro demonstration that Munc18 and tomosyn compete for syntaxin binding (Fujita et al., 1998). That the enhanced tethering observed in tom-1 mutants reflects an increase in UNC-18/ closed syntaxin dimers is further supported by the observation that in tom-1;unc-18 double mutants, vesicle tethering remains close to the reduced levels of unc-18 single mutants.

We have previously demonstrated that increased synaptic vesicle docking in tom-1 mutants reflects enhanced priming (Gracheva et al., 2006). Therefore, we assume that the improvement in vesicle priming observed in tom-1;unc-18 double mutants represents removal of inhibitory TOM-1/SNARE complexes, thereby permitting partial rescue of the primed vesicle pool even in the absence of UNC-18. The fact that priming remains defective relative to wildtype and much reduced relative to tom- 1 single mutants in these double mutants supports the hypothesis that UNC-18 also plays an important role in the priming stage of vesicle maturation. This conclusion is well supported by the recent literature, implicating Munc18-1 in fusion through interactions with the assembled SNARE complex (Gulyas-Kovacs et al., 2007; Deak et al., 2009). That UNC-18-dependent fusion is not merely a consequence of upstream tethering defects can be concluded from the analysis of open syntaxin mutants, which exhibit reduced tethering but accumulate docked vesicles, and exhibit robust release (Richmond et al., 2001). Whether TOM-1 normally aids in the transition from tethered to fully primed vesicles through the formation of tomosyn SNARE complexes remains to be determined.

In summary, we have identified two molecularly distinct stages in synaptic vesicle targeting that lead to the sequential tethering and docking of synaptic vesicles. Our data indicate that UNC-18 and closed syntaxin tether vesicles close to the plasma membrane upstream of priming. The transition to a docked and primed state, requires molecular rearrangements allowing syntaxin to adopt an open conformation compatible with SNARE complex formation, with which UNC-18 interacts to promote vesicle fusion competence. Tomosyn regulates both tethering and priming steps through competition with UNC-18 for syntaxin binding. These data support a growing body of literature implicating SM proteins and syntaxin in sequential stages of vesicle maturation, and suggest that similar mechanisms are employed by both synaptic vesicles and dense core vesicles (Toonen et al., 2006; Gulyas-Kovacs et al., 2007; Khvotchev et al., 2007; de Wit et al., 2009). We believe that inconsistencies in the field likely stem from methodological differences rather than cell type or species differences in these aspects of the exocytic cycle.

\section{ACKNOWLEDGMENTS}

We would like to thank; Dr. Erik Jorgensen for kindly providing the mosaic syntaxin strain EG3278, the C. elegans knockout consortium for tom-1 (ok285), Linda Juarez and Kristina Jarosius (RRC EM facility at UIC), Jay Campbell at UW-Madison for technical assistance with the high-pressure freeze EM, and Szi-Chieh Yu for assistance with data compilation. Funding for this study was provided by the National Institutes of Health (NIH) RO1 NS41477 and RO1 MH073156 to Janet E Richmond.

\section{REFERENCES}

Aravamudan, B., Fergestad, T., Davis, W. S., Rodesch, C. K., and Broadie, K. (1999). Drosophila UNC-13 is essential for synaptic transmission. Nat. Neurosci. 2, 965-971.

Augustin, I., Rosenmund, C., Sudhof, T. C., and Brose, N. (1999). Munc13-1 is essential for fusion competence of glutamatergic synaptic vesicles. Nature 400, 457-461.

Basu, J., Shen, N., Dulubova, I., Lu, J., Guan, R., Guryev, O., Grishin, N. V., Rosenmund, C., and Rizo, J. (2005). A minimal domain responsible for Munc13 activity. Nat. Struct. Mol. Biol. 12, 1017-1018.

Betz, A., Okamoto, M., Benseler, F., and Brose, N. (1997). Direct interaction of the rat unc-13 homologue Munc13-1 with the $\mathrm{N}$ terminus of syntaxin. $J$. Biol. Chem. 272:25202526.

Bouwman, J., Maia, A. S., Camoletto, P. G., Posthuma, G., Roubos, E. W., Oorschot, V. M., Klumperman, J., and Verhage, M. (2004). Quantification of synapse formation and maintenance in vivo in the absence of synaptic release. Neuroscience 126, 115-126.

Brenner, S., (1974). The genetics of Caenorhabditis elegans. Genetics 77, 71-94.

Broadie, K., Prokop, A., Bellen, H. J., O'Kane, C. J., Schulze, K. L., and Sweeney, S. T. (1995). Syntaxin and synaptobrevin function downstream of vesicle docking in Drosophila. Neuron 15, 663-673.

Burkhardt, P., Hattendorf, D. A., Weis, W. I., and Fasshauer, D. (2008). Munc18a controls SNARE assembly through its interaction with the syntaxin N-peptide. EMBO J. 27, 923-933.

de Wit, H., Cornelisse, L. N., Toonen, R. F., and Verhage, M. (2006). Docking of secretory vesicles is syntaxin dependent. PLoS ONE 1, e126. doi:10.1371/ journal.pone.0000126.

de Wit, H., Walter, A. M., Milosevic, I., Gulyas-Kovacs, A., Riedel, D., Sorensen, J. B., and Verhage, M. (2009). Synaptotagmin-1 docks secretory vesicles to syntaxin-1/SNAP-25 acceptor complexes. Cell 138, 935-946.

Deak, F., Xu, Y., Chang, W. P., Dulubova, I., Khvotchev, M., Liu, X., Sudhof, T. C., and Rizo, J. (2009). Munc18-1 binding to the neuronal SNARE complex controls synaptic vesicle priming. J. Cell Biol. 184, 751-764.
Dulubova, I., Khvotchev, M., Liu, S., Huryeva, I., Sudhof, T. C., and Rizo, J. (2007). Munc18-1 binds directly to the neuronal SNARE complex. Proc. Natl. Acad. Sci. U.S.A. 104, 2697-2702.

Dulubova, I., Sugita, S., Hill, S., Hosaka, M., Fernandez, I., Sudhof, T. C., and Rizo, J. (1999). A conformational switch in syntaxin during exocytosis: role of munc18. EMBO J. 18, 4372-4382.

Featherstone, D., and Broadie, K. (2002). Response: meaningless minis? Trends Neurosci. 25, 386-387.

Fujita, Y., Shirataki, H., Sakisaka, T., Asakura, T., Ohya, T., Kotani, H., Yokoyama, S., Nishioka, H., Matsuura, Y., Mizoguchi, A., Scheller, R. H., and Takai, Y. (1998). Tomosyn: a syntaxinlbinding protein that forms a novel 
complex in the neurotransmitter release process. Neuron 20, 905-915.

Gerber, S. H., Rah, J. C., Min, S. W., Liu, X., de Wit, H., Dulubova, I., Meyer, A. C., Rizo, J., Arancillo, M., Hammer, R. E., Verhage, M., Rosenmund, C., and Sudhof, T. C. (2008). Conformational switch of syntaxin-1 controls synaptic vesicle fusion. Science 321, 1507-1510.

Gladycheva, S. E., Lam, A. D., Liu, J., D’Andrea-Merrins, M., Yizhar, O., Lentz, S. I., Ashery, U., Ernst, S. A., and Stuenkel, E. L. (2007). Receptormediated regulation of tomosynsyntaxin 1A interactions in bovine adrenal chromaffin cells. J. Biol. Chem. 282, 22887-22899.

Gracheva, E. O., Burdina, A. O., Holgado, A. M., Berthelot-Grosjean, M., Ackley, B. D., Hadwiger, G., Nonet, M. L., Weimer, R. M., and Richmond, J. E. (2006). Tomosyn inhibits synaptic vesicle priming in Caenorhabditis elegans. PLoS Biol. 4, e261. doi:10.1371/ journal.pbio.0040261.

Gracheva, E. O., Burdina, A. O., Touroutine, D., Berthelot-Grosjean, M., Parekh, H., and Richmond, J. E. (2007). Tomosyn negatively regulates CAPS-dependent peptide release at Caenorhabditis elegans synapses. J. Neurosci. 27, 10176-10184.

Gulyas-Kovacs, A., de Wit, H., Milosevic, I., Kochubey, O., Toonen, R., Klingauf, J., Verhage, M., and Sorensen, J. B. (2007). Munc18-1: sequential interactions with the fusion machinery stimulate vesicle docking and priming. J. Neurosci. 27, 8676-8686.

Hammarlund, M., Palfreyman, M. T., Watanabe, S., Olsen, S., and Jorgensen, E. M. (2007). Open syntaxin docks synaptic vesicles. PLoS Biol. 5, e198. doi: 10.1371/journal.pbio.0050198.

Harrison, S. D., Broadie, K., van de Goor, J., and Rubin, G. M. (1994). Mutations in the Drosophila Rop gene suggest a function in general secretion and synaptic transmission. Neuron 13, 555-566.

Hatsuzawa, K., Lang, T., Fasshauer, D., Bruns, D., and Jahn, R. (2003). The R-SNARE motif of tomosyn forms SNARE core complexes with syntaxin 1 and SNAP-25 and down-regulates exocytosis. J. Biol. Chem. 278, 31159-31166.

Jahn, R., and Scheller, R. H. (2006). SNAREs-engines for membrane fusion. Nat. rev. 7, 631-643.

Jorgensen, E. M., Hartwieg, E., Schuske, K., Nonet, M. L., Jin, Y., and Horvitz, H. R. (1995). Defective recycling of synaptic vesicles in synaptotagmin mutants of Caenorhabditis elegans. Nature 378, 196-199.
Karatekin, E., Tran, V. S., Huet, S., Fanget, I., Cribier, S., and Henry, J. P. (2008). A 20-nm step toward the cell membrane preceding exocytosis may correspond to docking of tethered granules. Biophys. J. 94, 2891-2905.

Khvotchev, M., Dulubova, I., Sun, J., Dai, H., Rizo, J., and Sudhof, T. C. (2007). Dual modes of Munc18-1/ SNARE interactions are coupled by functionally critical binding to syntaxin-1 N terminus. J. Neurosci. 27, 12147-12155.

Liu, H., Dean, C., Arthur, C. P., Dong, M., and Chapman, E. R. (2009). Autapses and networks of hippocampal neurons exhibit distinct synaptic transmission phenotypes in the absence of synaptotagmin I. J. Neurosci. 29, 7395-7403.

Madison, J. M., Nurrish, S., and Kaplan, J. M. (2005). UNC-13 interaction with syntaxin is required for synaptic transmission. Curr. Biol. 15, 2236-2242.

McEwen, J. M., and Kaplan, J. M. (2008). UNC-18 promotes both the anterograde trafficking and synaptic function of syntaxin. Mol. Biol. Cell 19, 3836-3846.

McEwen, J. M., Madison, J. M., Dybbs, M., and Kaplan, J. M. (2006). Antagonistic regulation of synaptic vesicle priming by Tomosyn and UNC-13. Neuron 51 , 303-315.

O’Connor, V., Heuss, C., De Bello, W. M., Dresbach, T., Charlton, M. P., Hunt, J. H., Pellegrini, L. L., Hodel, A., Burger, M. M., Betz, H., Augustine, G. J., and Schafer, T. (1997). Disruption of syntaxinmediated protein interactions blocks neurotransmitter secretion. Proc. Natl. Acad. Sci. U.S.A. 94, 12186-12191.

Pobbati, A. V., Razeto, A., Boddener, M., Becker, S., and Fasshauer, D. (2004). Structural basis for the inhibitory role of tomosyn in exocytosis. J. Biol. Chem. 279, 47192-47200.

Reist, N.E., Buchanan, J., Li, J., DiAntonio, A., Buxton, E. M., and Schwarz, T. L. (1998). Morphologically docked synaptic vesicles are reduced in synaptotagmin mutants of Drosophila. J. Neurosci. 18, 7662-7673.

Richmond, J., (2009). Dissecting and recording from the C. Elegans neuromuscular junction. J. Vis. Exp. 24, 1165.

Richmond, J. E., Davis, W. S., and Jorgensen, E. M. (1999). UNC-13 is required for synaptic vesicle fusion in C. elegans. Nat. Neurosci. 2, 959-964.

Richmond, J. E., Weimer, R. M., and Jorgensen, E. M. (2001).An open form of syntaxin bypasses the requirement for UNC-13 in vesicle priming. Nature 412, 338-341.

Rickman, C., Medine, C. N., Bergmann,A., and Duncan, R. R. (2007). Functionally and Spatially Distinct Modes of munc18-Syntaxin 1 Interaction. J. Biol. Chem. 282, 12097-12103.

Rodkey, T. L., Liu, S., Barry, M., and McNew, J. A. (2008). Muncl8a scaffolds SNARE assembly to promote membrane fusion. Mol. Biol. Cell 19, 5422-5434.

Rostaing, P., Weimer, R. M., Jorgensen, E. M., Triller, A., and Bessereau, J. L. (2004). Preservation of immunoreactivity and fine structure of adult C. elegans tissues using high-pressure freezing. J. Histochem. Cytochem. 52, $1-12$.

Rowe, J., Calegari, F., Taverna, E., Longhi, R., and Rosa, P. (2001). Syntaxin 1A is delivered to the apical and basolateral domains of epithelial cells: the role of munc-18 proteins. J. Cell. Sci. 114, 3323-3332.

Sakisaka, T., Yamamoto, Y., Mochida, S., Nakamura, M., Nishikawa, K., Ishizaki, H., Okamoto-Tanaka, M., Miyoshi, J., Fujiyoshi, Y., Manabe, T., and Takai, Y. (2008). Dual inhibition of SNARE complex formation by tomosyn ensures controlled neurotransmitter release. J. Cell Biol. 183, 323-337.

Shen, J., Tareste, D. C., Paumet, F., Rothman, J. E., and Melia, T. J. (2007). Selective activation of cognate SNAREpins by Sec1/Munc18 proteins. Cell 128, 183-195.

Siksou, L., Rostaing, P., Lechaire, J. P., Boudier, T., Ohtsuka, T., Fejtova, A., Kao, H. T., Greengard, P., Gundelfinger, E. D., Triller, A., and Marty, S. (2007) Three-dimensional architecture of presynaptic terminal cytomatrix. J. Neurosci. 27, 6868-6877.

Siksou, L., Varoqueaux, F., Pascual, O. Triller, A., Brose, N., and Marty, S. (2009). A common molecular basis for membrane docking and functional priming of synaptic vesicles. Eur. J. Neurosci. 30, 49-56.

Stevens, D. R., Wu, Z. X., Matti, U., Junge, H. J., Schirra, C., Becherer, U., Wojcik, S. M., Brose, N., and Rettig, J. (2005). Identification of the minimal protein domain required for priming activity of Munc13-1. Curr. Biol. 15, 2243-2248.

Sudhof, T. C., (2004). The synaptic vesicle cycle. Annu. Rev. Neurosci. 27, 509-547.

Toonen, R. F., Kochubey, O., de Wit, H. Gulyas-Kovacs, A., Konijnenburg, B., Sorensen, J. B., Klingauf, J., and Verhage, M. (2006). Dissecting docking and tethering of secretory vesicles at the target membrane. EMBO J. 25, 3725-3737.

Varoqueaux, F., Sigler, A., Rhee, J. S., Brose, N., Enk, C., Reim, K., and Rosenmund, C. (2002). Total arrest of spontaneous and evoked synaptic transmission but normal synaptogenesis in the absence of Munc13-mediated vesicle priming. Proc. Natl. Acad. Sci. U.S.A. 99, 9037-9042.

Verhage, M., Maia, A. S., Plomp, J. J., Brussaard, A. B., Heeroma, J. H., Vermeer, H., Toonen, R. F., Hammer, R. E., van den Berg, T. K., Missler, M., Geuze, H. J., and Sudhof, T. C. (2000). Synaptic assembly of the brain in the absence of neurotransmitter secretion. Science 287, 864-869.

Voets, T., Toonen, R. F., Brian, E. C., de Wit, H., Moser, T., Rettig, J., Sudhof, T. C., Neher, E., and Verhage, M. (2001). Munc18-1 promotes large dense-core vesicle docking. Neuron 31, 581-591.

Weimer, R. M., Gracheva, E. O., Meyrignac, O., Miller, K. G., Richmond, J. E., and Bessereau, J. L. (2006). UNC-13 and UNC-10/rim localize synaptic vesicles to specific membrane domains. J. Neurosci. 26, 8040-8047.

Weimer, R. M., Richmond, J. E., Davis, W. S., Hadwiger, G., Nonet, M. L., and Jorgensen, E. M. (2003). Defects in synaptic vesicle docking in unc-18 mutants. Nat. Neurosci. 6, 1023-1030.

Yizhar, O., Matti, U., Melamed, R., Hagalili, Y., Bruns, D., Rettig, J., and Ashery, U. (2004). Tomosyn inhibits priming of large dense-core vesicles in a calciumdependent manner. Proc. Natl. Acad. Sci. U.S.A. 101, 2578-2583.

Zilly, F. E., Sorensen, J. B., Jahn, R., and Lang, T. (2006). Munc18-bound syntaxin readily forms SNARE complexes with synaptobrevin in native plasma membranes. PLoS Biol. 4, e330. doi:10.1371/journal.pbio.0040359.

Conflict of Interest Statement: The authors declare that the research was conducted in the absence of any commercial or financial relationships that could be construed as a potential conflict of interest.

Received:08 June 2010; paperpending published: 08 July 2010; accepted: 13 August 2010; published online: 05 October 2010. Citation: Gracheva EO, Maryon EB, Berthelot-Grosjean $M$ and Richmond JE(2010) Differential regulation of synaptic vesicle tethering and docking by UNC-18 and TOM-1. Front. Syn. Neurosci. 2:141. doi:10.3389/fnsyn.2010.00141

Copyright (C) 2010 Gracheva, Maryon, Berthelot-Grosjean and Richmond. This is an open-access article subject to an exclusive license agreement between the authors and the Frontiers Research Foundation, which permits unrestricted use, distribution, and reproduction in any medium, provided the original authors and source are credited. 